Special issue of the 3rd International Conference on Computational and Experimental Science and Engineering (ICCESEN 2016)

\title{
Signal Processing Deployment in Power Quality Disturbance Detection and Classification
}

\begin{abstract}
F.Z. DEKHANDJI*
Laboratory Signals and Systems, Institute of Electrical and Electronic Engineering, University M'hamed Bougara of Boumerdes, Av. de l'Indépendance, 35000 Boumerdes, Algeria

Power quality disturbances have adverse impacts on the electric power supply as well as on the customer equipment. Therefore, the detection and classification of such problems is necessary. In this paper, a fast detection algorithm for power quality disturbances is presented. The proposed method is a hybrid of two algorithms, abc-0dq transformation and $90 \circ$ phase shift algorithms. The proposed algorithm is fast and reliable in detecting most voltage disturbances in power systems such as voltage sags, voltage swells, voltage unbalance, interrupts, harmonics, etc. The three-phase utility voltages are sensed separately by each of the algorithms. These algorithms are combined to explore their individual strengths for a better performance. When a disturbance occurs, both algorithms work together to recognize this distortion. This control method can be used for critical loads protection in case of utility voltage distortion. Simulation and analysis results obtained in this study illustrate high performance of the strategy in different single-phase and three-phase voltage distortions.
\end{abstract}

DOI: 10.12693/APhysPolA.132.415

PACS/topics: power quality, detection, classification, hybrid algorithms, reliability

\section{Introduction}

Electric power quality has emerged as a major area of electric power engineering. The predominant reason for this emergence is the increase in the sensitive end-use equipment. The term power quality is defined as: the concept of powering and grounding sensitive equipment in a matter that is a suitable to the operation of the equipment [1-5]. The term power quality is however a misnomer. It is actually the voltage quality that is being addressed. Power is the rate of energy delivery and is proportional to the product of voltage and current. It is difficult to define the quality of this quantity in a meaningful manner. The power supply system can control only the voltage quality; it has no control over the currents that particular loads might draw [3].

A common characteristic of most electronic loads is that they are sensitive to voltage variations. Computers and other sensitive loads can lower their performance or even shut down the process they are in control due to those variations. Voltage variations can be classified as disturbances that produce voltages below the nominal value, which are called voltage sags, and disturbances that produce voltages above the nominal value, which are called voltage swells [4]. The Information Technology Industry Council (ITIC) has published a curve showing a voltage envelope that can be tolerated by most information technology equipment [6-9]. This curve is a reference widely used to define when the supply of equipment needs to be compensated due to whether voltage sag or a voltage swells. On every existing approach to compen-

\footnotetext{
*e-mail: fzdekhandji@yahoo.fr
}

sate for voltage disturbances, the first step is to detect the disturbance itself, and then other actions will come such as compensation or disconnection [7, 8].

The importance of the detection stage is critical for applications like static transfer switches (STS) and uninterruptible power supplies (UPS), or approaches where the utility supply must be disconnected as part of the compensation. Statistics shows that STS can transfer critical loads from utility supply to ancillary supply within $1 / 4$ cycle, but critical loads can only tolerate voltage disturbances for a maximum of $1 / 2$ cycle [6]. However, by means of a fast algorithm, it is possible to recognize voltage disturbances and send the trip signal to STS within less than $1 / 4$ cycles after the disturbance. This would enable the critical loads, such as hospitals, telecommunication companies, hotels, etc., to continue their operation without any interruption [10-16].

Several approaches have been reported on detection, localization, and classification of voltage distortions. Examples of these methods are the Fourier transforms, notch filter, or band pass filters, $p-q$ theory via the $\alpha-\beta$ transformation, extended phase-locked loop (PLL) methods, and wavelet transform algorithm [7]. Each of these approaches could detect voltage disturbances, but then each has its drawbacks. For example, the Fourier transform and PLL are too slow in returning typical tracking information, and wavelet transform usually returns results that can be difficult to interpret [8]. Further, the IEEE has proposed abc-0dq transformation algorithm as a voltage disturbance recognizer. The most important advantage of this algorithm is its simplicity and its ability to detect different voltage disturbances very quickly; however, this algorithm is unable to recognize balanced three-phase voltage DC offset disturbance. Further, it takes a long time to recognize unbalanced disturbances such as single-phase-to-earth fault. Meanwhile, analysis 
of other algorithms results shows that $90^{\circ}$ phase shift algorithm is able to detect balanced three-phase DC offset and many unbalanced voltage disturbances very fast. Another important advantage of this algorithm is its ability to recognize fault types and their characteristics [9]. Subsequently, by combining abc-0dq transformation algorithm and $90^{\circ}$ phase shift algorithm, a fast and accurate algorithm to recognize all voltage disturbances and faults characteristics can be achieved. This explains the underlying principle behind the proposed algorithm in this paper [10, 11, 17-19].

\section{Disturbance detection algorithms}

\subsection{The abc-0dq transformation theory}

The abc-0dq transformation converts a three-phase signal, consisting of three different vectors into two vectors in a two-dimensional frame of reference (see Fig. 1). The two axes are called the direct axis and the quadrature axis. This theory is usually employed in the control of induction motors, known as field orientated control (FOC). Here, the complex three-phase induction motor can be modelled as a DC motor by performing simple transformations known as abc-0dq transformation.

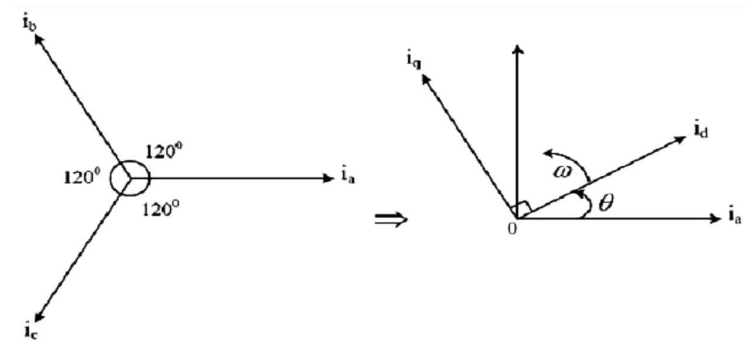

Fig. 1. abc-0dq transformation.

The relationship between abc and 0dq variables for the machine shown in Fig. 2 was defined by the Park transformation. This relationship is given in Eq. (1):

$$
v_{0 d q}=[P] v_{a b c},
$$

where the transformation matrix

$$
[P]=\sqrt{\frac{2}{3}}\left[\begin{array}{ccc}
\frac{1}{\sqrt{2}} & \frac{1}{\sqrt{2}} & \frac{1}{\sqrt{2}} \\
\cos \theta & \cos \left(\theta-\frac{2}{3} \pi\right) & \cos \left(\theta+\frac{2}{3} \pi\right) \\
\sin \theta & \sin \left(\theta-\frac{2}{3} \pi\right) & \sin \left(\theta+\frac{2}{3} \pi\right)
\end{array}\right]
$$

and $\theta$ is the angle of the direct axis with respect to the aphase - the reference axis. Interestingly the q-axis is at an angle of $90^{\circ}$ to the direct axis, and both are rotating with the same angular velocity $\omega$ as the sinusoidal phase quantities [6].

\section{2. $90^{\circ}$ phase shift algorithm}

This section analyses the principle of work using phaseshifting method to detect power quality (PQ) disturbances based on the Hilbert transform (HT), a brief overview of the HT is given in the following subsection.

\subsubsection{Basic principle of the $H T$}

The HT of the function $x(t)$ is defined as the convolution between $x(t)$ and $h(t)=\frac{1}{\pi t}$. The convolution expression is [20]:

$$
\begin{aligned}
& \boldsymbol{H}(x(t))=x^{h}(t)=x(t) * h(t)=x(t) * \frac{1}{\pi t}= \\
& \quad \frac{1}{\pi} \int_{-\infty}^{+\infty} \frac{x(\tau)}{t-\tau},
\end{aligned}
$$

where $*$ represents the convolution, $\boldsymbol{H}(x(t))$ and $x^{h}(t)$ represent a HT in time-domain. Different from other transform methods, the HT of time-domain function is still in time-domain. In frequency-domain, the HT can express as

$$
\boldsymbol{X}^{h}(f)=X(f) H(f) .
$$

Because the Fourier transform (FT) of $h(t)$,

$$
\boldsymbol{F}(h(t)) \boldsymbol{H}(f)=-\mathrm{j} \operatorname{sgn}(f)=-\mathrm{j}\left\{\begin{array}{rr}
1 & f<0, \\
-1 & f>0,
\end{array}\right.
$$

where sgn is the signum function $[2-5,9,20]$, so multiplying the negative frequency of the $x(f)$ by $\mathrm{j}$ and multiplying the positive frequency of $x(f)$ by $-\mathrm{j}$ one can obtain HT in frequency-domain. The HT result can be deduced from FT, so

$$
x^{h}(t)=x(t) * h(t)=\boldsymbol{F}^{-1}(X(f) H(f)) .
$$

As input signal $x(t)=\sin \left(2 \pi f_{i} t\right)$, its FT is

$$
\boldsymbol{X}(f)=\frac{\mathrm{j}}{2}\left(\delta\left(f+f_{i}\right)-\delta\left(f-f_{i}\right)\right)
$$

and multiplying the negative frequency of the $x(f)$ by $\mathrm{j}$, and multiplying positive frequency of the $x(f)$ by $-\mathrm{j}$ :

$$
\begin{gathered}
\boldsymbol{X}^{h}(f)=\frac{1}{2}\left(-\delta\left(f+f_{i}\right)-\delta\left(f-f_{i}\right)\right)= \\
\boldsymbol{F}\left(-\cos \left(2 \pi f_{i} t\right)\right),
\end{gathered}
$$

do the Fourier inversion to both sides of the formula (8):

$$
\boldsymbol{H}\left(\sin \left(2 \pi f_{i} t\right)\right)=-\cos \left(2 \pi f_{i} t\right) .
$$

Equation (9) shows that HT of the sine function is the negative cosine function, the result also shows that it makes $-\mathrm{j}$ phase-shifting to the input signal. The same argument, if input signal is the cosine function, the HT of the function is the sine function, the result shows that it makes $-\mathrm{j}$ phase-shifting to the input signal, too. So the HT can provide $90^{\circ}$ phase-shifting and does not influence the amplitude of the frequency-spectrum components.

\subsection{The hybrid algorithm}

This algorithm is a hybrid of two algorithms, abc-0dq transformation and $90^{\circ}$ phase shift algorithms. The hybrid algorithm detects the disturbance and then issues a trip signal to enable subsequent actions mitigation or protection. The abc-0dq transformation algorithm is based on the theory that allows a set of three-phase voltages to be represented as DC voltages in a $\mathrm{d}-\mathrm{q}$ synchronous rotating frame. Thus, any disturbance at the utility input voltages will be detected as a disturbance in the $d-q$ frame. Hence, in the positive sequence the component of the line voltages $V_{+}$can be obtained as follows: 


$$
V_{+}=\sqrt{V_{d}^{2}+V_{q}^{2}}
$$

In the $90^{\circ}$ phase shift algorithm, the utility line voltages are converted to DC quantities and compared with reference line voltage $\left(V_{\text {ref }}\right)$, which is the nominal voltage of the supply. These comparisons give error signals shown as er (abc-0dq transformation algorithm), $\mathrm{er}_{1}, \mathrm{er}_{2}$ and $\mathrm{er}_{3}$ of the three phases $A, B$, and $C$, respectively $\left(90^{\circ}\right.$ phase shift algorithm). When the disturbance begins, both algorithms are able to detect the voltage disturbance and send a trip signal to their output at two different times. By an OR gate, only the first trip signal will be given as the hybrid algorithm's output for rapid recognition of the voltage disturbance. This method classifies fault types and their characteristics such as amplitude, frequency, time duration.

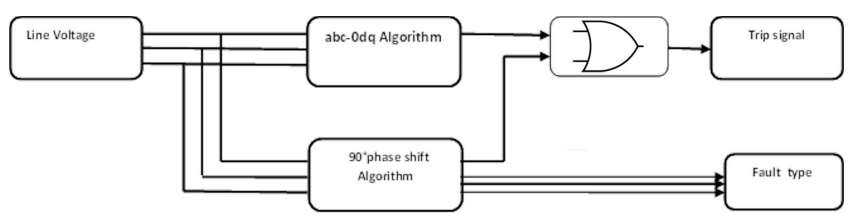

Fig. 2. The block diagram of the hybrid algorithm.

(a)

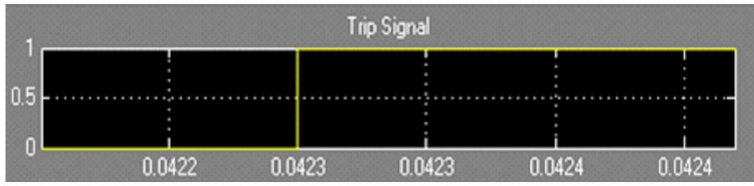

(b)

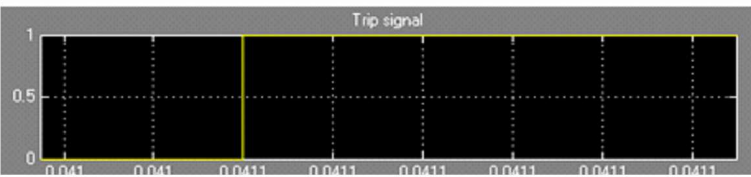

(c)

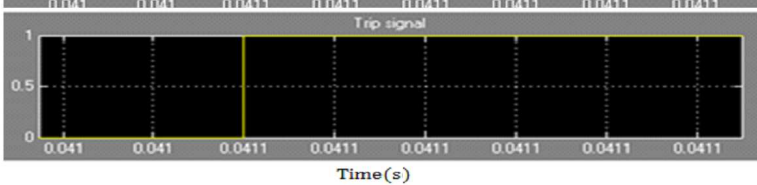

Fig. 3. Detection of starting times of the voltage disturbance: (a) abc-0dq transformation, (b) $90^{\circ}$ phase shift algorithm, (c) the hybrid algorithm.

\section{Simulation results and discussions}

The work starts by loading PQ disturbance signals such as voltage swells, voltage sags, voltage interruption and harmonics into the PQ disturbances detection system. Next, the simulation results consist in generating a trip signal to detect the disturbance occurrence and an error signal to classify the disturbance type.

\subsection{Voltage sags}

The ideal input voltage is

$$
x(t)=A \sin \left(w_{0} t+\varphi_{0}\right) .
$$

Assuming $A=1$, and $\varphi_{0}=0$ then $x(t)=\sin \left(w_{0} t\right)$.
For voltage sags case, the input voltage formula will be as follows:

$$
x(t)=\left(1-\alpha\left(u\left(t-t_{1}\right)-u\left(t-t_{2}\right)\right)\right) \sin \left(w_{0} t\right) .
$$

In the formula $(11), u(t)$ is the unit step function, function, $\alpha=0.1-0.9$ is the voltage sags amplitude; $1 \mathrm{~T}<$ $t_{2}-t_{1}<9 \mathrm{~T}$ is the voltage sags duration, $t_{1}$ is the voltage sags initial time, $t_{2}$ is the end time.

Simulation results show that a $50 \%$ voltage sag created on phase $A$ took place when $t$ was between 0.04 and $0.08 \mathrm{~s}$. The $90^{\circ}$ phase-shifting disturbance detection system can detect the time when input voltage sags take place, and accurately detect sags amplitude, initial time, end time and duration. Figure $3 \mathrm{a}-\mathrm{c}$ shows the trip signals of the voltage disturbance detectors. The $90^{\circ}$ phase shift algorithm detects the sag at $0.0411 \mathrm{~s}$, while abc0dq algorithm detects the same fault after a delay time of $0.0023 \mathrm{~s}$. The hybrid algorithm determines the instance of the fault at $0.0411 \mathrm{~s}$. Figure 4 shows the error signal of the phase $A$ voltage.

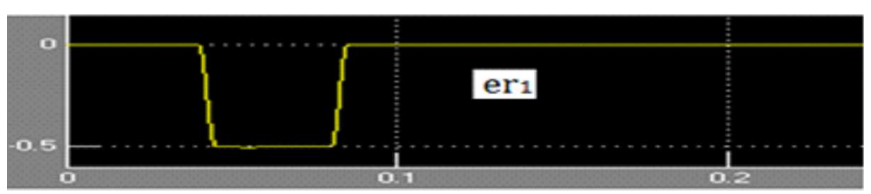

Fig. 4. Error signals for a 0.5 per unit (pu) voltage sag on phase $A$.

\subsection{Voltage swell}

For voltage swells case, the input voltage formula will be as follows:

$$
x(t)=\left(1+\alpha\left(u\left(t-t_{1}\right)-u\left(t-t_{2}\right)\right)\right) \sin \left(w_{0} t\right) .
$$

In the formula (12), $u(t)$ is the unit step function, $\alpha=$ $0.1-0.9$ is the voltage swells amplitude, $1 \mathrm{~T}<t_{2}-t_{1}<$ $9 \mathrm{~T}$ is the voltage swells duration, $t_{1}$ is the voltage swells initial time, $t_{2}$ is the end time.

The basic functions of the proposed system are based on the comparison of a voltage reference and the measured terminal voltage $\left(V_{a}, V_{b}, V_{c}\right)$ which gives the error voltage signal.

(a)

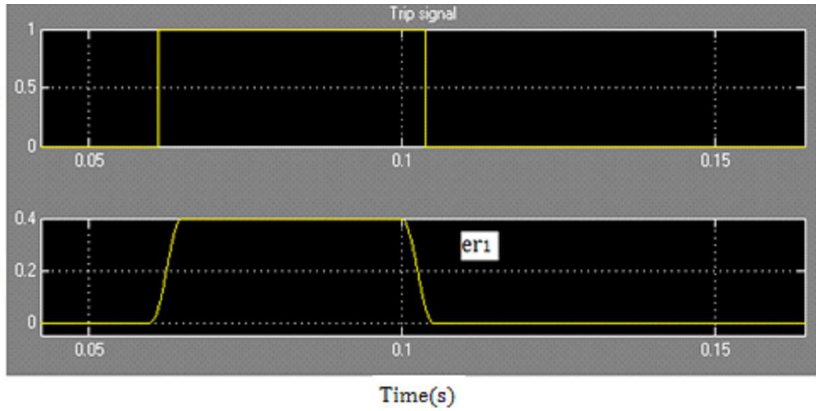

Fig. 5. Error signal for a 0.4 pu voltage swell on phase A. (a) Trip signal, (b) error signal. 
The voltage swell is detected when the supply increases up to $25 \%$ of the reference value. The error signal is used to detect the fault type. Figure 5 shows the simulation results of a voltage swell of amplitude $0.4 \mathrm{pu}$ initiated on phase $A$ at $0.06 \mathrm{~s}$ and cleared at $0.1 \mathrm{~s}$.

\subsection{Harmonics}

The general expression for harmonics is

$$
\begin{aligned}
& x(t)=A\left(\alpha_{1} \sin \left(w_{0} t\right)+\alpha_{3} \sin \left(3 w_{0} t\right)+\alpha_{5} \sin \left(5 w_{0} t\right)\right. \\
& \left.\quad+\alpha_{7} \sin \left(7 w_{0} t\right)\right) .
\end{aligned}
$$

Assuming $A=1$, and $\alpha_{1}=1$ then for voltage harmonics case, the input voltage formula will be as follows:

$$
\begin{aligned}
& x(t)=\sin \left(w_{0} t\right)+\alpha_{3} \sin \left(3 w_{0} t\right)+\alpha_{5} \sin \left(5 w_{0} t\right) \\
& \quad+\alpha_{7} \sin \left(7 w_{0} t\right)(13) .
\end{aligned}
$$

In the formula (13) $\alpha_{3}, \alpha_{5}$, and $\alpha_{7}$ are the 3rd, 5th, and 7 th harmonic amplitude, as the higher order harmonics are small, over here it can be neglected.

A three-phase utility voltage distorted by the fifth harmonic of $0.5 \mathrm{pu}$ amplitude between 0.05 and $0.1 \mathrm{~s}$ was applied to the proposed voltage disturbance detection algorithm. Simulation results in Fig. 6 show the $90^{\circ}$ phase shift algorithm. As could be seen, Fig. 5b detects voltage harmonic disturbance through the trip signal at $0.0505 \mathrm{~s}$ and abc-0dq algorithm, (c) detects the harmonic voltages at $0.0554 \mathrm{~s}$ and the hybrid algorithm, (d) shows the occurrence of the harmonics after a time delay of $0.0005 \mathrm{~s}$. Figure 5e shows the error signal, where the utility line voltage is distorted by the 5 th harmonic with $0.5 \mathrm{pu}$ amplitude and approximately a frequency of 5 freq $\mathrm{Hz}$ which is $250 \mathrm{~Hz}$ (the line freq $=50 \mathrm{~Hz}$ ).

(a)

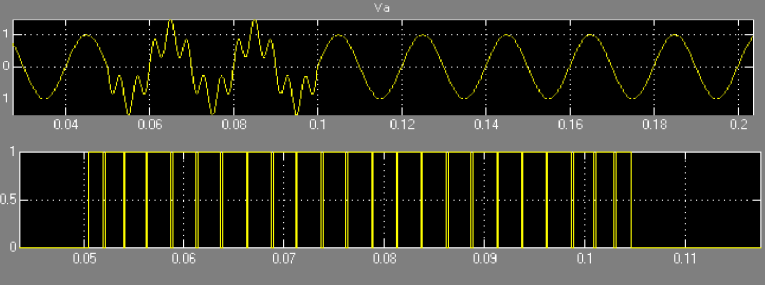

(c)

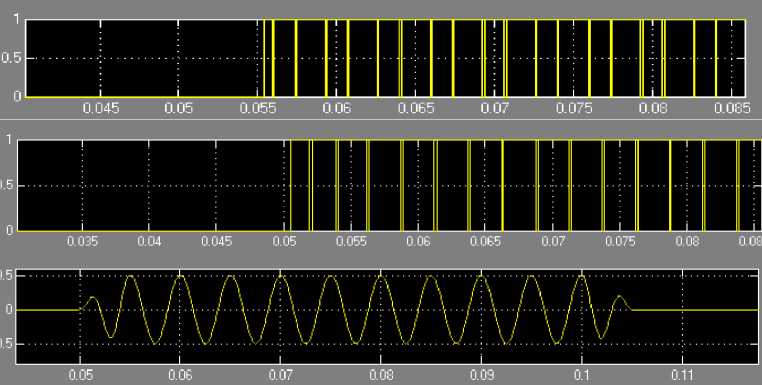

Fig. 6. Detection times comparison of algorithms.(a) Single-phase voltage distorted by the 5th harmonic, (b) $90^{\circ}$ phase shift algorithm, (c) abc-0dq algorithm, (d) hybrid algorithm, and (e) error signal.

\subsection{Interruption}

An interruption is said to have occurred when the supply voltage decreases to less than 0.1 per unit. Figure 7 shows a momentary interruption initiated on phase $A$ at $0.03 \mathrm{~s}$ and cleared at $1.6 \mathrm{~s}$.

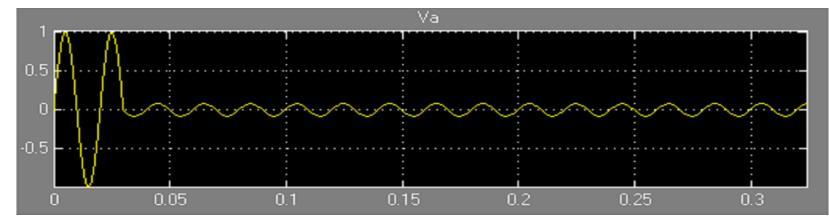

Fig. 7. Phase $A$ distorted by a momentary $0.08 \mathrm{pu}$ interruption.

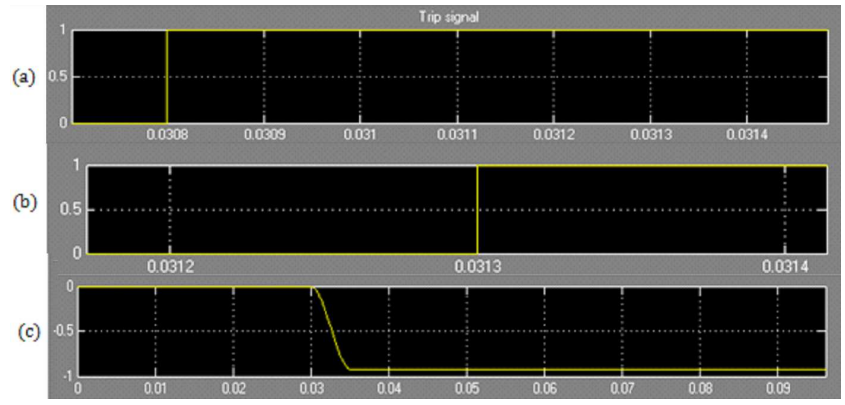

Fig. 8. Detection times comparison fault characteristic for $0.08 \mathrm{pu}$ interruption. (a) $90^{\circ}$ phase shift algorithm, (b) abc-0dq algorithm, (c) error signal.

Simulation results show that $90^{\circ}$ phase shift algorithm detects the fault in $0.0308 \mathrm{~s}$ with time delay of $0.0008 \mathrm{~s}$, while the abc-0dq algorithm detects the disturbance in 0.0313 with $0.0013 \mathrm{~s}$ time delay. Figure 8 displays detection times and fault characteristic for the occurred interruption.

\section{Conclusions}

A hybrid algorithm has been used for the detection and classification of various voltage disturbances in power systems. This algorithm combines abc-0dq transformation technique and $90^{\circ}$ phase shift method, by exploring the strengths of each of the techniques. The simulation of different voltage disturbances such as sags, voltage swells, voltage harmonics, and interruption has been done. The presented results demonstrate the effectiveness of the proposed method such that the worst case detection time for all types of disturbances is less than $1 / 4$ cycle.

\section{References}

[1] Harjit Singh Birdi, Ph.D. Thesis, University of Saskatchewan, Canada 2006.

[2] M.H.J. Bollen, Understanding Power Quality Problems, Wiley IEEE Press, 2000.

[3] P. Pillay, M. Manyage, IEEE Power Eng. Rev. 5, 10 (2001).

[4] R.P. Bingham, Sags and Swells, Technology and Products Dranetz-BM, 1998. 
[5] R.C. Dugan, M.F. McGranaghan, S. Santoso, H.W Beaty, Electrical Power Systems Quality, 3rd ed., McGraw-Hill, 2012.

[6] E. Pouresmaeil, M.F. Akorede, Euro. Trans. Electr. Power 21, 555 (2011).

[7] Z. Chen, M. Wei, in: The Third Int. Conf. on Electric Utility Deregulation and Restructuring and Power Technologies (DRPT 2008), Nanjing (China), 2008.

[8] O. Poisson, P. Rioual, M. Meunier, IEEE Trans. Power Deliv. 15, 1039 (2000).

[9] Chen Chunling, Yang Yong, Power Quality Disturbances Detection Based on Hilbert Phase-Shifting, Asia-Pacific Power and Energy Engineering Conference, 2009, School of Information and Electric Engineering, Shenyang Agricultural University, 2009.

[10] F.Z. Dekhandji, L. Refoufi, in: Progress in Clean Energy, Vol. II, Novel Systems and Applications, Springer, 2015, p. 711.

[11] F.Z. Dekhandji, L. Refoufi, H. Bentarzi, Int. J. System Assurance Eng. Manage. 8, 393 (2015).
[12] M. Imal, Acta Phys. Pol. A 130, 245 (2016).

[13] R. Kiliç, Acta Phys. Pol. A 130, 487 (2016).

[14] O.M. Pişirira, O. Bingöl, Acta Phys. Pol. A 130, 36 (2016).

[15] Z. Er, Acta Phys. Pol. A 130, 72 (2016).

[16] D. Reddy Edla, V. Gondlekar, V. Gauns, Acta Phys. Pol. A 130, 78 (2016).

[17] J. Schlabbach, D. Blume, Voltage Quality in Electrical Power Systems, Institution of Electric Engineers, London 2001.

[18] Mir Ahmad Koolaiyan, in: Proc. 8th WSEAS Int. Conf. on Electric Power Systems, Venice 2008.

[19] R. Naidoo, P. Pillay, IEEE Trans. Power Deliv. 22, 1056 (2007)

[20] M. Johansson, M.Sc. Thesis, Växjo University, Sweden. 